\title{
Bladder Lymphangioma Treated by Holmium Laser: Extremely Rare Case Report
}

\author{
Asaad Moradi', Babak Kazemzadeh Azad ${ }^{2}$, Mahyar Fasihi' ${ }^{3}$, Fereshteh Aliakbari \\ ${ }^{1}$ Department of Urology, Firozgar Hospital, Iran University of Medical Sciences, Tehran, Iran \\ ${ }^{2}$ Men's Health \& Reproductive Health Research Center, Shahid Beheshti University of Medical Sciences, Tehran, Iran \\ ${ }^{3}$ Department of Urology, Hasheminejad Hospital, Iran University of Medical Sciences, Tehran, Iran
}

\author{
*Correspondence to \\ Babak Kazemzadeh Azad, Men's \\ Health \& Reproductive Health \\ Research Center, Shahid Beheshti \\ University of Medical Sciences, \\ Tehran, Iran. \\ Tel/Fax: +98 2122712234 \\ Cell phone: +98 9111863352 \\ Email: bab1412000@yahoo.com. \\ Published online March 15, 2020

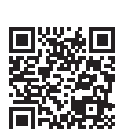

\begin{abstract}
Introduction: Lymphangioma is a sporadic benign tumor of the bladder. It is a congenital disorder and based on the size of lymphatic spaces, it is divided into 3 types of capillary, cavernous, and cystic.

Case Report: In this paper, we presented a 40-year-old woman with microscopic hematuria and a normal urinary ultrasound. Urethrocystoscopy showed a flat $4 \mathrm{~mm}$ highlighted strawberry-like lesion on the right lateral wall of the bladder. After a cold cup biopsy, the lesion was coagulated by the holmium: YAG (Ho: YAG) laser.

Conclusion: In Bladder Lymphangioma Based on the size of the lesion, partial cystectomy or minimally invasive surgeries such as laser modality would be the principal treatment.

Keywords: Lymphangioma; Bladder tumor; Laser; Ho-YAG.
\end{abstract}

\section{Introduction}

Lymphangioma is a congenital benign tumor of lymphoid tissue that occurs due to abnormal drainage of lymph vessels and lymph tissue proliferation. This tumor does not repulse after treatment. Lymphangioma is divided into three types of capillary, cavernous, and cystic based on the size of lymphatic spaces. ${ }^{1,2}$ Lymphangioma symptoms appear once they become large enough to lead to complications such as infection, rupture, or torsion. Typically, cystic lymphoid is formed in the neck and auxiliary with a prevalence of $70 \%$ and $20 \%$ respectively, and it is rarely seen in the urinary system. ${ }^{3,4}$

The holmium laser has been used in urology since 1993 with a wavelength of $2140 \mathrm{~nm}$ absorbed by the tissue fluid. This property of helium leads to vaporization without deep coagulation and provides a safe margin. ${ }^{5}$ Holmium: YAG (Ho: YAG) has good penetration depth, which helps in staunching the flow of blood; it is also used for other urologic surgeries, including $\mathrm{BPH}$, urolithiasis, bladder tumor, genital skin lesion and urethrotomy. ${ }^{6-8}$

Lymphangioma is an extremely rare disease of the bladder and has been reported only in three cases in the world. To our knowledge, this patient treated by Ho: YAG is the fourth case who has been reported since 1983.

\section{Case Report}

The patient was a 40-year-old woman presented with alternative microscopic hematuria without irritative urinary symptoms and dysuria in the last three months. Urine analysis and urine culture were normal at the time of admission. Although she did not file a complaint of gross hematuria, she had a pale sclera, and her hemoglobin level was $8 \mathrm{~g} / \mathrm{dL}$. Considering the anemia and microscopic hematuria, further examinations were requested. Urinary ultrasound was insignificant. In a pelvic computed tomography (CT) scan, we noticed calcification around the hip area, which seemed to be relevant to uterine myoma; no calcification was found in the kidneys, ureters, and bladder.

For further investigation, the patient underwent urethrocystoscopy. A flat $4 \mathrm{~mm}$ highlighted strawberrylike lesion was seen on the right lateral wall of the bladder. The cold cup biopsy was done, and the lesion was coagulated by the Ho: YAG laser. The Ho: YAG laser was applied with a $365-\mu \mathrm{m}$ fiber at a setting of $2 \mathrm{~J}$ and a frequency of 10 to $20 \mathrm{~Hz}$. The patient was discharged in good condition. In the pathology, the histological section showed large lymphatic channels lined by flattened endothelium in loose connective tissue underlying transitional epithelium of the bladder, which indicated lymphangioma of the bladder.

\section{Discussion}

Intra-abdominal lymphangioma in adults is quite rare, and few cases of bladder lymphangioma have been reported. These lesions are usually quiescent ${ }^{9,10}$ but can 
mimic bladder carcinomas. Lymphangiomas mainly affect the head and neck; most are diagnosed before the age of five. ${ }^{3,4}$

While the most common symptom of bladder tumors is painless hematuria, they are mostly transitional and less commonly squamous cell carcinomas.

To the authors' knowledge, only three cases of bladder lymphangioma have been reported all over the world since 1983. The first case was in a child, started from the wall of the bladder and invaded the peritoneal cavity through the bladder wall. The patient had painless macroscopic hematuria which continued for two months before hospitalization. Treatment consisted of partial cystectomy. ${ }^{11,12}$ The second case was a 49-year-old man, admitted because of irritative urinary symptoms. In his cystoscopy, a papillary mass was observed between two holes. It was removed by transurethral resection. After 3 months, all voiding symptoms were improved, and follow-up cystoscopy was normal. ${ }^{13}$

The third case was an 8-year-old girl who was examined in 2010. She had hematuria as well as long-lasting intermittent fever. The tumor appeared as a shiny red mass in cystoscopy, and a bulge into the bladder on the right lateral wall was detected by ultrasound and CT. A partial cystectomy was done, and the pathological analysis indicated bladder lymphangioma. There was no evidence of recurrence in her three-year follow-up. ${ }^{12}$

Two cases underwent partial cystectomy, and transurethral resection was performed for the third patient. None of them recurred. Since the size of the lesion was small in our patient, the whole abrasion was removed by the Ho: YAG laser. Our patient was the only case whose bladder lymphangioma was cured by minimally invasive treatment. In a two-year follow-up with cystoscopy, there was not any recurrence.

\section{Conclusion}

Although lymphangioma in the bladder is rare, it might be a reason for microscopic hematuria and should be considered. Radiography and ultra-sound results can be negative; thus, the best method of diagnosis is cystoscopy. Based on the size of the lesion, partial cystectomy or minimally invasive surgeries such as laser modality would be the principal treatment.

\section{Ethical Considerations}

Written consent was obtained from the patient for the promotion of knowledge.

\section{Conflict of Interests}

The authors declare no conflict of interest.

\section{Funding}

None.

\section{Acknowledgments}

The authors wish to acknowledge the assistance of the Urology operation room staff.

\section{References}

1. Wilson SR, Bohrer S, Losada R, Price AP. Retroperitoneal lymphangioma: an unusual location and presentation. $J$ Pediatr Surg. 2006;41(3):603-5. doi: 10.1016/j. jpedsurg. 2005.11. 057.

2. Liu H, Luo $\mathrm{CH}$. Retroperitoneal Lymphangioma. In: Luo CH, ed. Retroperitoneal Tumors. Dordrecht: Springer; 2018. p. 215-221. doi: 10.1007/978-94-024-1167-6_23.

3. Hancock BJ, St-Vil D, Luks FI, Di Lorenzo M, Blanchard H. Complications of lymphangiomas in children. $J$ Pediatr Surg. 1992;27(2):220-226. doi: 10.1016/00223468(92)90316-Y.

4. Minocha PK, Roop L, Persad R. Cases of atypical lymphangiomas in children. Case Rep Pediatr. 2014;2014:626198. doi.org/10.1155/2014/626198

5. Razzaghi MR, Fallah Karkan M, Ghiasy S, Javanmard B. Laser application in iran urology: a narrative review. J Lasers Med Sci. 2018;9(1):1-6. doi:10.15171/jlms.2018.01.

6. Abedi AR, Razzaghi MR, Allameh F, Aliakbari F, FallahKarkan M, Ranjbar A. Pneumatic Lithotripsy Versus Laser Lithotripsy for Ureteral Stones. J Lasers Med Sci. 2018;9(4):233-6. doi:10.15171/jlms.2018.42.

7. Fallah karkan M, Razzaghi MR, Karami H, Ghiasy S, Tayyebiazar A, Javanmard B. Experience of 138 transurethral urethrotomy with Holmium:YAG Laser. J Lasers Med Sci. 2019;10(2):104-107. doi:10.15171/ jlms.2019.17.

8. Ghiasy S, Fallah-Karkan M, Razzaghi MR, Ranjbar A, Rahavian A, Javanmard B. Is Holmium Laser an appropriate modality to treat genital warts? J Lasers Med Sci. 2019;10(1):70-74. doi:10.15171/jlms.2019.11.

9. Mahle C, Schwartz M, Popek E, Bocklage T. Intraabdominal lymphangiomas in children and adults: assessment of proliferative activity. Arch Pathol Lab Med. 1997;121(10):1055-62.

10. Muramori K, Zaizen Y, Noguchi S. Abdominal lymphangioma in children: report of three cases. Surg Today. 2009;39(5):414-17. doi: 10.1007/s00595-0083854-z.

11. Bolkier M, Ginesin Y, Lichtig C, Levin DR. Lymphangioma of bladder. J Urol. 1983;129(5):1049-1050. doi.org/10.1016/ S0022-5347(17)52536-7.

12. Niu ZB, Yang Y, Hou Y, Chen H, Liu X, Wang CL. Lymphangioma of bladder. Urology. 2010;76(4):955-7. doi: 10.1016/j.urology.2010.03.050.

13. Wyler SF, Bachmann A, Singer G, Gasser TC, Sulser T. First case of lymphangioma of the bladder in an adult. Urol Int. 2004;73(4):374-5. doi: 10.1159/000082242. 